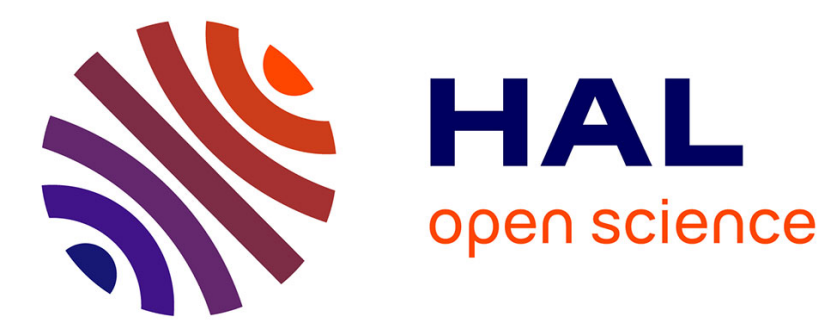

\title{
Estimation of depth on thick edges from sharp and blurred images
}

Christophe Simon, Frédérique Bicking, Thierry Simon

\section{To cite this version:}

Christophe Simon, Frédérique Bicking, Thierry Simon. Estimation of depth on thick edges from sharp and blurred images. IEEE IMTC conference, May 2002, Anchorage, United States. pp.323-328. hal-00333010

\section{HAL Id: hal-00333010 https://hal.science/hal-00333010}

Submitted on 22 Oct 2008

HAL is a multi-disciplinary open access archive for the deposit and dissemination of scientific research documents, whether they are published or not. The documents may come from teaching and research institutions in France or abroad, or from public or private research centers.
L'archive ouverte pluridisciplinaire HAL, est destinée au dépôt et à la diffusion de documents scientifiques de niveau recherche, publiés ou non, émanant des établissements d'enseignement et de recherche français ou étrangers, des laboratoires publics ou privés. 


\title{
Estimation of depth on thick edges from sharp and blurred images
}

\author{
Christophe Simon Frederique Bicking and Thierry Simon
}

\begin{abstract}
This article deals with the generalization of a local depth estimation method using sharp edges and blurred edges. This Depth from Defocus method is explained and the theoretical relations are defined. Improvements concerning the generalization and the noise sensitivity on the depth estimation are developed and application conditions are exposed. Some results on synthetic images are presented to illustrate the method efficiency.
\end{abstract}

Index Terms-depth map, depth from defocus, thick edge, 3D primitives

\section{INTRODUCTION}

The depth, distance between the visible surface of objects in a scene and the sensor of the camera, is a useful indication for the computation of coordinates of the points belonging to this surface in a 3D-space of reference. Many methods have been developed to obtain these coordinates. Some techniques, appointed Depth From Focus (DFF) ([1], $[2],[3],[4])$, search for patches of sharpness in the image and link them to the depth by classic relationships of the geometrical optic. They present the major disadvantage of a long computing time to obtain a depth map. Other methods called Depth From Defocus (DFD) ([5], [6], [7], [8]), use the perceptible optical blur on heterogeneous image patch corresponding to edges or textures by using at least two images of the same scene with only one point of view. These images are acquired with different parameters adjustments of the optical system. Our technique uses only sharp and blurred images of the same scene. The optical blur, characteristic of the depth, is observed on gray level discontinuities in the blurred image.

In DFD methods, a relationship between the depth, the parameters of the camera and the optical blur in images is searched. The physical ef-

Ecole Supérieure des Sciences et Technologies de l'Ingénieur de Nancy

Université Henri Poincaré Nancy 1, 2 Rue Jean Lamour, 54519 Vandœuvre-lès-Nancy Cedex, France

Phone: (+33) 383-501634, email:

Christophe.Simon@esstin.uhp-nancy.fr

Ecole Supérieure des Sciences et Technologies de l'Ingénieur de Nancy

Université Henri Poincaré Nancy 1, 2 Rue Jean Lamour, 54519 Vandœuvre-lès-Nancy Cedex, France

Phone: (+33) 383-501634, email:

Frederique.Bicking@esstin.uhp-nancy.fr

IUT de Figeac

Avenue de Nayrac, 46100 Figeac, France

Phone: $\quad(+33) \quad 565-503068$,

Simon.IUT.Figeac@wanadoo.fr fect produced by the modification of the aperture of the diaphragm on images characteristics is used in order to establish this relationship. In the image formation process, to an image plane with a fixed distance and for a scene containing several depth planes, only one plane gives a sharp or a focused image. Points of objects belonging to the other planes will form spots more or less blurred according to their distance to the image plan. The formation of the optical blur is linked to the optical transfer function of the system ([9]) in the spatial domain represented by its Point Spread Function (PSF). Thus, a convolution relationship (1) is established between the sharp image $I_{n}(i, j)$, the blurred image $I_{f}(i, j)$ and the $P S F(i, j)$ where $i$ and $j$ are the coordinates of a pixel and $\underset{2 D}{\otimes}$ is the $2 \mathrm{D}$ convolution operator.

$$
I_{f}(i, j)=I_{n}(i, j) \underset{2 D}{\otimes} \operatorname{PSF}(i, j)
$$

The PSF depends on the properties of the optic materials (indication of refraction) and on the geometrical form of the lens (focal distance) as well as on the parameters shot (distance of the plan object to the main plan, aperture, lighting).

A realistic model taking into account both the aspects of the geometrical optic effects of the diffraction and the defaults of lens does not exist. Several models have been proposed and we have selected the model of distribution of luminance given by a $2 \mathrm{D}$-gaussian with a spread parameter $\sigma_{s_{o}}$ corresponding to a depth $s_{o}(2)$.

$$
P S F_{s_{o}}(i, j)=\frac{1}{2 \pi \sigma_{s_{o}}^{2}} e^{-\frac{i^{2}+j^{2}}{2 \sigma_{s_{o}}^{2}}}
$$

Thanks to a calibration procedure, values of $\sigma_{s_{o}}$ are directly associated to depths $s_{O}$ by estimation of the constants $c$ and $m$ that are characteristic of a set of camera tuning parameters $([10])$ :

$$
\frac{1}{s_{o}}=\frac{\sigma_{s_{o}}}{m}-\frac{c}{m}
$$

This article deals with the generalization of a local depth estimation on edges. First of all the estimation method of the amount of blur linked with the depth using a couple of focused and defocused images is explained. More developments can be found in ([11]). The theoretical relations are exposed and improvement with introduction of a generalized form is described. Therefore, a new 
relation to compute the spread parameter on several points belonging to the edges is defined and allows to reduce the noise sensitivity of the method. Conditions of application are pointed out and lead to new consideration such as constraints for further development. Finally, some experiments are carried out to highlight improvements of noise sensitivity, importance of application constraints and performances. Results on synthetic images are then presented.

\section{INITIAL METHOD AND THEORETICAL IMPROVEMENTS}

Classic depth estimation approaches use either the spatial content of the image by geometrical characteristics ([12], [5]) as well as the form of objects in the scene ([1]), or the frequency information by a Fourier analysis ([7]).

Discontinuities of luminance expressed on the edges of observed objects for which the blur effects are easily perceptible, are used. The method is similar to those proposed by Pentland where the acquisition of a sharp image with a closed aperture and a blurred image with an open aperture is retained. The position of edges is detected with the Prewitt operator. An estimation of the optical blur is obtained from the module of the gradients of gray levels. With the ratio of sharp and blurred images magnitudes, it is possible to identify the spread parameter $\sigma_{s_{o}}$ and thus to estimate the depth $s_{o}$ with the relation (3).

This ratio can be expressed with the relation (4) where $\left|\nabla I_{n}(i, j)\right|$ and $\left|\nabla I_{f}(i, j)\right|$ are respectively the sharp image gradient magnitude and the blurred image gradient magnitude.

$$
R(i, j)=\frac{\left|\nabla I_{n}(i, j)\right|}{\left|\nabla I_{f}(i, j)\right|}
$$

Let the sharp image $I_{n}(i, j)$ present gray level discontinuities in slope with a magnitude $(b-a)$ and a length $\varepsilon$ under a single direction $(\theta= \pm k \pi / 2)$. In other direction, the sharp image does not present gray level variations. The gray level function for the sharp edge profile $c_{n}(x)$ can be expressed by (5) where $x$ is graduated in pixel and represents one line or one column of this image:

$$
c_{n}(x)= \begin{cases}a & x<x_{0} \\ a+\left(\frac{b-a}{\varepsilon}\right)\left(x-x_{0}\right) & x_{0} \leq x \leq x_{0}+\varepsilon \\ b & x>x_{0}+\varepsilon\end{cases}
$$

In order to compute $\nabla I_{n}(i, j)$, the Prewitt operator $(\nabla p)$ is used.

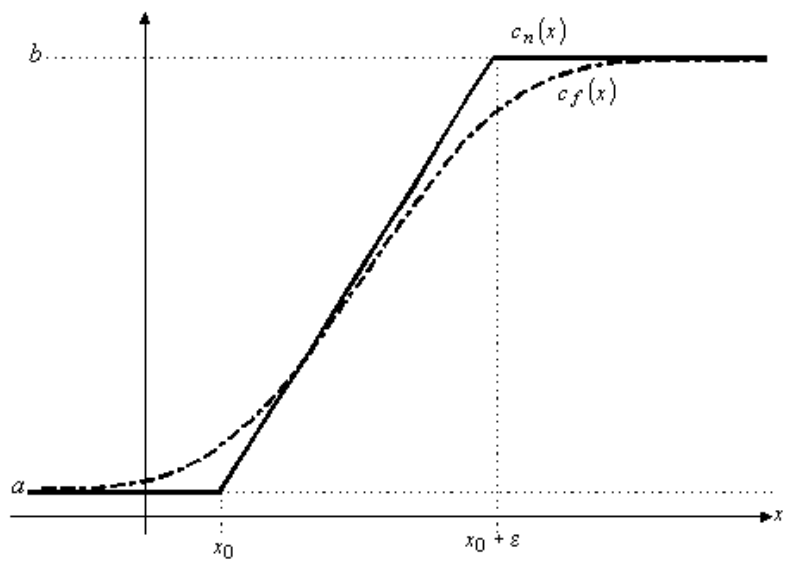

Fig. 1. Sharp and blurred edge profiles

$$
\begin{aligned}
\nabla_{p} I_{n}(i, j)= & {\left[\left(\frac{1}{3}\left[\begin{array}{lll}
1 & 0 & -1 \\
1 & 0 & -1 \\
1 & 0 & -1
\end{array}\right] \underset{2 D}{\otimes} I_{n}(i, j)\right)^{2}+\right.} \\
& \left.\left(\frac{1}{3}\left[\begin{array}{lll}
-1 & -1 & -1 \\
0 & 0 & 0 \\
1 & 1 & 1
\end{array}\right] \underset{2 D}{\left.\stackrel{\otimes}{D} I_{n}(i, j)\right)^{2}}\right]^{\frac{1}{2}} 6\right)
\end{aligned}
$$

For the direction $\theta=0 \pm k \pi, I_{n}(i, j-1)=$ $I_{n}(i, j)=I_{n}(i, j+1)$ and (6) becomes equivalent to $(7)$ :

$$
\left|\nabla_{p} I_{n}(i, j)\right|=\left|\left[\begin{array}{lll}
1 & 0 & -1
\end{array}\right] \underset{2 D}{\stackrel{\otimes}{D}} I_{n}(i, j)\right|
$$

For the direction $\theta=\pi / 2 \pm k \pi, I_{n}(i-1, j)=$ $I_{n}(i, j)=I_{n}(i+1, j)$ and (6) becomes equivalent to (8):

$$
\left|\nabla_{p} I_{n}(i, j)\right|=\left|\left[\begin{array}{l}
-1 \\
0 \\
1
\end{array}\right] \underset{2 D}{\otimes} I_{n}(i, j)\right|
$$

Thus, for the direction $\theta= \pm k \pi / 2$, the 2D-1D correspondence allows to use:

$$
\left|\nabla_{p} c_{n}(x)\right|=\left|c_{n}(x) \otimes\left[\begin{array}{lll}
-1 & 0 & 1
\end{array}\right]\right|
$$

where $\otimes$ is the 1D convolution operator. With this 2D-1D correspondence between the used image and the sharp edge profile, the blurred edge profile is obtained by the relation (10):

$$
c_{f}(x)=c_{n}(x) \otimes P_{s_{o}}(x)
$$

where $P_{s_{o}}(x)$ is the monodimensional form of the point spread function expressed by: $P_{s_{o}}(x)=$ $\frac{1}{\sqrt{2 \pi} \sigma_{s_{o}}} e^{-\frac{x^{2}}{2 \sigma_{s_{o}}^{2}}}$. Sharp and blurred edge profiles are represented on figure 1. The gradient magnitude for the blurred image is given by (11): 


$$
\left|\nabla_{p} c_{f}(x)\right|=\left|\nabla_{p} c_{n}(x) \otimes P S F(x)\right|
$$

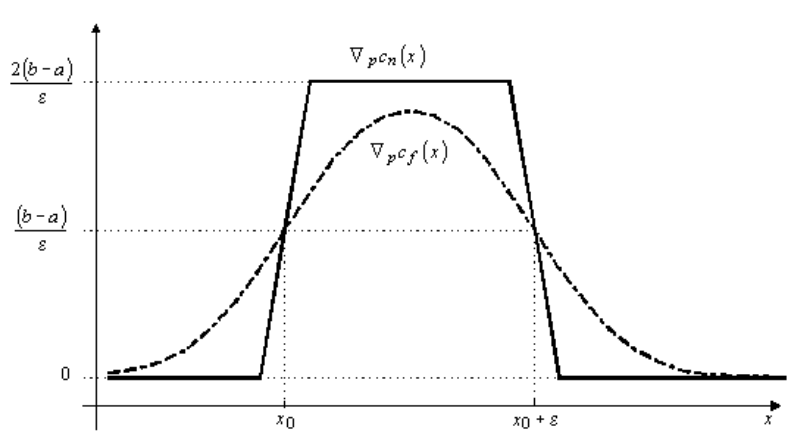

Fig. 2. Sharp and blurred edge gradient profiles

The ratio given by (4) can be computed for several values of $\varepsilon$ at two particular points $x_{0}$ and $x_{0}+\varepsilon$ with $R\left(x_{0}\right)=R\left(x_{0}+\varepsilon\right)$ :

For $\varepsilon=1, R\left(x_{0}\right)=\frac{\sigma_{s_{o}} \sqrt{2 \pi}}{1+\exp \left(\frac{-1}{2 \sigma_{s_{o}}^{2}}\right)}$

For $\varepsilon=2, R\left(x_{0}\right)=\frac{\sigma_{s_{o}} \sqrt{2 \pi}}{1+2 \exp \left(\frac{-1}{2 \sigma_{s_{o}}^{2}}\right)+\exp \left(\frac{-4}{2 \sigma_{s_{o}}^{2}}\right)}$

For $\varepsilon \geq 3, R\left(x_{0}\right)=\frac{\sigma_{s_{o}} \sqrt{2 \pi}}{1+2 \exp \left(\frac{-1}{2 \sigma_{s_{o}}^{2}}\right)+2 \exp \left(\frac{-4}{2 \sigma_{s_{o}}^{2}}\right)}$ $+\exp \left(\frac{-9}{2 \sigma_{s_{O}}^{2}}\right)$

These relations linking the spread parameter $\sigma_{s_{o}}$ to the ratio value are numerically solved. The ratio computed at the point $x_{0}$ allows to find one value for $\sigma_{s_{o}}$ and to obtain a value of the depth $s_{o}$ by the relation (3).

Previous works ([11],[13]) use a maximum length $\varepsilon$ set to 3 , considering that no larger values occur with optics of high quality. Using standard quality, edges of length $\varepsilon$ higher than 3 can be found. Thus, it appears necessary to have specific relations of the ratio $R\left(x_{0}\right)$ for each value of $\varepsilon$. To avoid a lot of relations, we propose a general expression of $R\left(x_{0}\right)$.

The gradient of the sharp edge is: $\nabla_{p} c_{n}(x)=$ $\left(\frac{b-a}{\varepsilon}\right)$ and the blurred one is: $\nabla_{p} c_{f}(x)=P_{s_{o}}(0)+$ $P_{s_{o}}(\varepsilon)+2 \sum_{u=1}^{\varepsilon-1} P_{s_{o}}(u)$. They are represented on figure 2 .

So, the general form of the ratio can be expressed by:

$$
R\left(x_{o}, \varepsilon\right)=\frac{1}{P_{s_{o}}(0)+P_{s_{o}}(\varepsilon)+2 \sum_{u=1}^{\varepsilon-1} P_{s_{o}}(u)}
$$

This relation allows to estimate the spread parameter $\sigma_{s_{o}}$ for the points $x_{0}$ and $x_{0}+\varepsilon$ from the values of the gradients magnitudes of the sharp and blurred images at these two points. In presence of noisy images, estimation will be corrupted. Thus, the use of each point belonging to the edge give several estimated values of $\sigma_{s_{o}}$. The estimation results will appear more robust.

The expression of the ratio for each point between $x_{0}$ and $x_{0}+\varepsilon$ is given by (14):

$$
\begin{aligned}
& R(x)=\frac{2}{P_{s_{o}}\left(x-x_{0}\right)+P_{s_{o}}\left(x-\left(x_{0}+\varepsilon\right)\right)} \\
& +2 \sum_{u=1}^{\varepsilon-1} P_{s_{o}}\left(x-\left(x_{0}+u\right)\right)
\end{aligned}
$$

To reduce noise sensitivity, and thus to improve the quality of the solution, $\varepsilon+1$ estimations of the spread parameter $\sigma_{s_{o}}$ are computed and a statistical value is attributed to the point $x_{0}$ in the depth map. That needs more computing time but the estimation obtained is better.

\section{EXPERIMENTAL RESULtS}

This paragraph deals with the results obtained by the proposed method. In a first stage, we illustrate the efficiency of the method with two examples based on synthetic images with different parameters. Next, a comparison between results obtained with the method applied only at the point $x_{0}$ and those obtained with the use of all points $x \in\left[x_{0}, x_{0}+\varepsilon\right]$ are presented. The noise sensitivity of the method is evaluated on noisy images with different signal to noise ratios (SNR) by comparing the estimated value to the true value of the spread parameter. Finally, the orientation constraint influence on estimation is shown by experiments with different orientation values.

\section{A. Blur estimation for different parameters}

In order to illustrate the efficiency of the proposed method, the estimation results for a synthetic image (fig.3a) blurred with a spread parameter $\sigma_{s_{o}}=3$ (fig.3b) are shown on figure 3c. For this image, the length of edges are fixed at $1(\varepsilon=1)$. As it can be seen, the estimation of $\sigma_{s_{o}}$ gives good results. However, estimation is corrupted for the center of the image. It can be explained by the mutual influence of closely related points. The nearest contour points have some contribution on the points in a small neighbourhood. The closer the edges are and the more spread the PSF is, the more overvalued the spread parameter will be.

Figure 4 shows the estimation results for an image with edges length fixed at $5(\varepsilon=5)$ and a spread parameter $\sigma_{s_{o}}=5$. As it can be seen, the method gives very good results with a total estimation error 


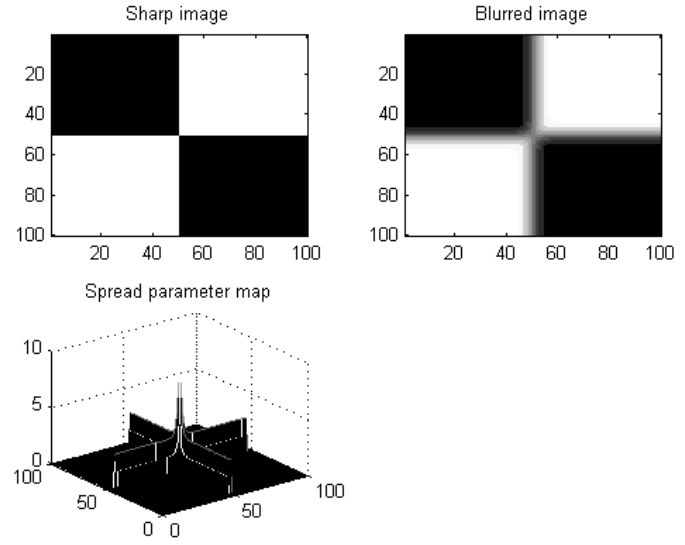

Fig. 3. Impact of closely related edges on the estimation of $\sigma_{s_{o}}=3$ and $\varepsilon=1$
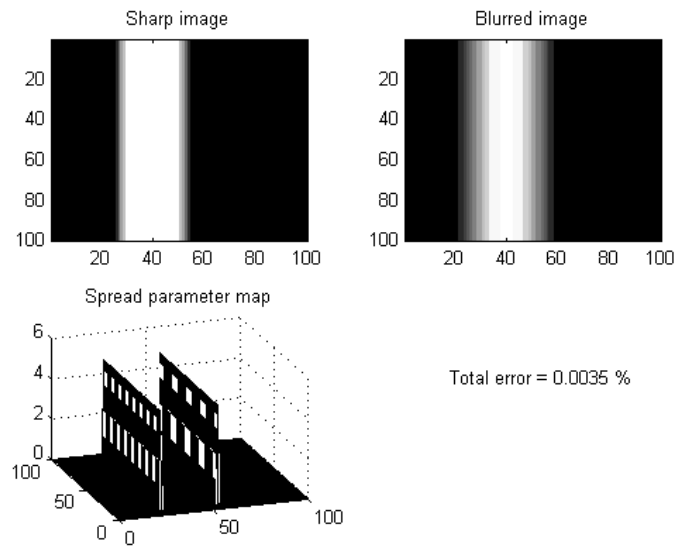

Total error $=0.0035 \%$

Fig. 4. Estimation results for $\varepsilon=5$ and $\sigma_{s_{o}}=5$

mean of $3.5 E^{-3 \%}$. In this case, the mutual influence of edges is not encountered. That's why such performances are obtained.

\section{B. Exploitation of each point belonging to the edge profile}

Results obtained with the method applied only at the point $x_{0}$ and those obtained with the use of all points $x \in\left[x_{0}, x_{0}+\varepsilon\right]$ are presented in tables I and II respectively. The method was tested on synthetic images presenting vertical discontinuities. As it can be seen, for small values of $\sigma_{s_{o}}$ and high values of $\varepsilon$, the estimation error is important. However, the improvement of the blur estimation using all points is significant in comparison with estimation results obtained using point $x_{0}$ only.

\section{Noise sensitivity}

To investigate the performance of the depth estimation method under noisy environment, we consider a number of low grade images. The noisy im-
TABLE I

ESTIMATION ERROR IN \% WITH THE METHOD APPLIED AT POINT $x_{o}$

\begin{tabular}{|c||c|c|c|c|c|c|c|c|}
\hline & $\sigma$ & & & & & & & \\
$\varepsilon$ & 0.5 & 0.8 & 1 & 1.5 & 2 & 3 & 5 & 8 \\
\hline \hline 1 & 2.8 & 0.0 & 0.0 & 0.0 & 0.0 & 0.0 & 0.0 & 0.0 \\
2 & 18.4 & 0.0 & 0.0 & 0.0 & 0.0 & 0.0 & 0.0 & 0.0 \\
3 & 42.9 & 0.1 & 0.0 & 0.0 & 0.0 & 0.0 & 0.0 & 0.0 \\
4 & 64.1 & 3.7 & 0.0 & 0.0 & 0.0 & 0.0 & 0.0 & 0.0 \\
5 & 82.7 & 14.2 & 0.0 & 0.0 & 0.0 & 0.0 & 0.0 & 0.0 \\
6 & 99.6 & 24.7 & 1.2 & 0.0 & 0.0 & 0.0 & 0.0 & 0.0 \\
7 & 115.1 & 34.4 & 7.5 & 0.0 & 0.0 & 0.0 & 0.0 & 0.0 \\
8 & 129.6 & 43.5 & 14.8 & 0.0 & 0.0 & 0.0 & 0.0 & 0.0 \\
9 & 143.2 & $52 . .1$ & 21.6 & 0.0 & 0.0 & 0.0 & 0.0 & 0.0 \\
10 & 156.1 & 60.1 & 28.0 & 14.6 & 0.0 & 0.0 & 0.0 & 0.0 \\
\hline
\end{tabular}

TABLE II

ESTIMATION ERROR IN \% WITH THE METHOD APPLIED AT ALL POINTS $x \in\left[x_{0}, x_{0}+\varepsilon\right]$

\begin{tabular}{|c|c|c|c|c|c|c|c|c|}
\hline$\varepsilon$ & $\begin{array}{c}\sigma \\
0.5\end{array}$ & 0.8 & 1 & 1.5 & 2 & 3 & 5 & 8 \\
\hline 1 & 2.8 & 0.0 & 0.0 & 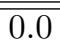 & 0.0 & 0.0 & 0.0 & 0.0 \\
\hline 2 & 13.2 & 0.0 & 0.0 & 0.0 & 0.0 & 0.0 & 0.0 & 0.0 \\
\hline 3 & 23.8 & 0.1 & 0.0 & 0.0 & 0.0 & 0.0 & 0.0 & 0.0 \\
\hline 4 & 31.2 & 1.5 & 0.0 & 0.0 & 0.0 & 0.0 & 0.0 & 0.0 \\
\hline 5 & 36.4 & 4.7 & 0.0 & 0.0 & 0.0 & 0.0 & 0.0 & 0.0 \\
\hline 6 & 42.1 & 7.1 & 0.3 & 0.0 & 0.0 & 0.0 & 0.0 & 0.0 \\
\hline 7 & 46.8 & 8.6 & 1.9 & 0.0 & 0.0 & 0.0 & 0.0 & 0.0 \\
\hline 8 & 51.9 & 10.1 & 3.2 & 0.0 & 0.0 & 0.0 & 0.0 & 0.0 \\
\hline 9 & 56.3 & 11.4 & 4.3 & 0.0 & 0.0 & 0.0 & 0.0 & 0.0 \\
\hline 10 & 61.1 & 13.1 & 5.1 & 2.6 & 0.0 & 0.0 & 0.0 & 0.0 \\
\hline
\end{tabular}

ages are generated by adding independently, identically distributed Gaussian noise to the noise-free images. The signal-noise-ratio (SNR) is computed for each image. The SNR is defined by the classical relation (15):

$$
S N R=10 \log \left(\frac{\overline{I_{n}^{2}(i, j)}}{n^{2}(i, j)}\right)
$$

It is assumed that the noisy sharp image and the noisy blurred image have the same SNR value.

The effect of noise is significant on image gray levels. In order to evaluate the images grade, we pay attention to the gray level variations $(\Delta G L)$ under SNR values. Table III shows this correspondence.

Performance comparison is based on a synthetic sharp image that presents vertical discontinuities. The estimation method was applied with respect 
TABLE III

Gray LEVEL VARIATION FOR DIFFERENT SIGNAL TO NOISE RATIO

\begin{tabular}{|c||c|c|c|c|c|c|}
\hline$\Delta \mathrm{GL}$ & 2 & 3 & 5 & 8 & 16 & 26 \\
\hline SNR & 55 & 50 & 45 & 40 & 35 & 30 \\
\hline
\end{tabular}

to different values of SNR, edge length and spread parameter.

TABLE IV

ESTIMATION ERROR IN \% FOR DIFFERENT VALUES OF SNR,

$\sigma$ AND $\varepsilon$

\begin{tabular}{|c|c||c|c|c|c|c|}
\hline & & SNR (dB) & & & & \\
$\sigma$ & $\varepsilon$ & 30 & 35 & 40 & 45 & 50 \\
\hline \hline 1 & 1 & 4.19 & 1.17 & 1.49 & 0.77 & 0.30 \\
& 2 & 7.91 & 6.44 & 1.98 & 1.08 & 0.11 \\
& 3 & 4.35 & 11.22 & 2.39 & 12.32 & 3.09 \\
& 4 & 1.65 & 18.86 & 3.94 & 11.52 & 3.48 \\
& 5 & 8.31 & 8.45 & 3.98 & 0.80 & 2.75 \\
& 6 & 21.79 & 16.46 & 34.96 & 15.74 & 8.37 \\
\hline 2 & 1 & 3.69 & 1.57 & 0.03 & 0.10 & 0.30 \\
& 2 & 2.08 & 2.11 & 0.20 & 0.32 & 0.36 \\
& 3 & 4.26 & 2.94 & 1.38 & 0.03 & 0.48 \\
& 4 & 17.34 & 2.06 & 2.31 & 0.96 & 0.60 \\
& 5 & 2.50 & 15.51 & 8.20 & 11.87 & 5.21 \\
& 6 & 7.54 & 12.47 & 9.42 & 8.25 & 7.13 \\
\hline 3 & 1 & 4.28 & 1.95 & 0.40 & 0.59 & 0.13 \\
& 2 & 0.84 & 0.76 & 0.78 & 0.30 & 0.20 \\
& 3 & 5.42 & 0.30 & 0.65 & 0.36 & 0.09 \\
& 4 & 4.55 & 3.10 & 0.92 & 1.02 & 0.32 \\
& 5 & 10.32 & 7.06 & 3.33 & 0.34 & 0.80 \\
& 6 & 9.51 & 4.61 & 1.19 & 0.53 & 0.87 \\
\hline
\end{tabular}

Table IV illustrates the resulting estimation error under noisy environment obtained by the proposed method. When the noise level is low, the performances of the method are satisfactory. With the increase of the noise level, the estimation error increases but remains still acceptable. Note that for a SNR value of 30 , the 26 gray level variation corresponds to a $10 \%$ variation on the gray levels of the image.

\section{Influence of edge orientation}

The relation (14) was defined thanks to the definition of the equivalence between the expression of the gradient image magnitude at $x_{0}$ and the edge profile at $x_{0}$ using the correspondence 2D-1D defined above. So, the relation (14) must be applied when the object contour in the image respects this equivalence established for a discontinuity orientation $\theta=0+n \frac{\pi}{2}$. Nevertheless, we also try to evalu- ate experimentally the orientation influence on the accuracy of the results in order to know if the relation (14) can be used for other orientations even if approximated evaluation of spread parameter and consequently error on depth estimation are generated. If the accuracy is not satisfactory the discontinuity orientation will be integrated as an application constraint.

TABLE V

ESTIMATION ERROR IN \% FOR DIFFERENT VALUES OF EDGES ORIENTATION (DEGREE), $\sigma$ AND $\varepsilon$

\begin{tabular}{|c|c||c|c|c|c|c|c|}
\hline$\%$ & & & & $\varepsilon$ & & & \\
\hline$\sigma_{s_{o}}$ & $\theta$ & 1 & 2 & 3 & 4 & 5 & 6 \\
\hline \hline 0.8 & 0 & 0.0 & 0.0 & 0.1 & 1.5 & 4.8 & 7.1 \\
& 10 & 0.3 & 6.9 & 5.9 & 9.6 & 17.4 & 23.8 \\
& 20 & 25.8 & 4.1 & 13.8 & 0.3 & 18.9 & 68.6 \\
& 30 & 30.7 & 1.1 & 12.0 & 0.2 & 33.1 & 51.4 \\
& 40 & 31.4 & 28.1 & 23.5 & 12.4 & 35.5 & 27.2 \\
& 45 & 24.4 & 24.4 & 23.6 & 21.4 & 29.3 & 21.8 \\
\hline 1 & 0 & 0.0 & 0.0 & 0.0 & 0.0 & 0.0 & 0.4 \\
& 10 & 4.1 & 26.3 & 10.4 & 0.2 & 6.3 & 26.1 \\
& 20 & 6.6 & 12.6 & 21.1 & 10.0 & 17.0 & 31.6 \\
& 30 & 7.6 & 20.1 & 20.2 & 9.8 & 13.5 & 31.9 \\
& 40 & 40.1 & 18.8 & 29.5 & 2.4 & 22.4 & 23.9 \\
& 45 & 35.7 & 33.8 & 28.8 & 28.5 & 35.1 & 30.3 \\
\hline 1.5 & 0 & 0.0 & 0.0 & 0.0 & 0.0 & 0.0 & 0.0 \\
& 10 & 8.0 & 29.8 & 17.9 & 10.8 & 3.9 & 0.0 \\
& 20 & 28.8 & 9.5 & 25.1 & 20.4 & 6.5 & 29.1 \\
& 30 & 39.7 & 11.9 & 24.3 & 19.1 & 15.9 & 8.3 \\
& 40 & 37.0 & 33.3 & 37.8 & 18.9 & 2.1 & 9.9 \\
& 45 & 34.5 & 35.1 & 32.7 & 29.5 & 41.6 & 35.3 \\
\hline 2 & 0 & 0.0 & 0.0 & 0.0 & 0.0 & 0.0 & 0.0 \\
& 10 & 8.0 & 31.5 & 14.0 & 12.0 & 10.7 & 6.0 \\
& 20 & 2.7 & 15.4 & 24.3 & 22.0 & 17.0 & 3.8 \\
& 30 & 35.1 & 19.0 & 23.5 & 26.3 & 2.9 & 2.5 \\
& 40 & 36.7 & & 28.6 & 21.5 & 8.4 & 15.9 \\
& 45 & 32.2 & 33.0 & 34.4 & 28.6 & 40.1 & 40.5 \\
\hline 3 & 0 & 0.0 & 0.0 & 0.0 & 0.0 & 0.0 & 0.0 \\
& 10 & 5.6 & 24.4 & 12.0 & 8.0 & 9.5 & 9.3 \\
& 20 & 21.1 & 12.2 & 19.9 & 19.8 & 14.3 & 4.5 \\
& 30 & 31.7 & 17.2 & 19.2 & 26.0 & 0.6 & 7.9 \\
& 40 & 37.8 & 26.6 & 30.7 & 22.9 & 11.0 & 23.3 \\
& 45 & 36.0 & 32.4 & 35.5 & 31.5 & 39.4 & 31.8 \\
\hline
\end{tabular}

Experiments on synthetic images have been carried out with some selected fixed edge directions $\theta$ (in degree) and different values of edge lengths $\varepsilon$ and spread parameters $\sigma_{s_{o}}$.

As it can be seen on table $\mathrm{V}$, when the edge orientation is $\theta=0$, the orientation condition is fullfilled and the performance is very satisfactory. On the contrary, when the edge orientation is moved away from the value 0 , the method presents important es- 
timation errors. So, we can conclude that the edge orientation is a meaningful application constraint and that the relation (14) will have to be adapted for other orientations.

\section{Conclusion}

A local depth perception method was presented by focusing on two important generalizations. On the one hand, mathematical relations were defined to allow blur estimation on thick edges. On the other hand, the theoretical improvement to estimate the depth from all points of the edge profile was defined. It allows to reduce the influence of noise on image data. In addition, we have shown that relationships allowing the estimation of the depth were valid only for orientations $\theta=0 \pm n \frac{\pi}{2}$. The respect of this constraint strongly reduces the number of points for blur estimation. In order to obtain a dense depth map, the expression of the ratio between the gradient magnitude of the sharp image and the gradient magnitude of the blur image has to be defined for other edge directions.

\section{REFERENCES}

[1] T. Darell and K. Wohn, "Depth from focus using a pyramid architecture," Pattern Recognition Letters, vol. 11 (12), pp. 787-796, 1990.

[2] P. Grossman, "Depth from focus," Pattern Recognition Letters, vol. 5, pp. 63-69, 1987.

[3] E. Krotkov, "Focusing," International Journal of Computer Vision, vol. 1, pp. 223-237, 1987.

[4] T. Choi M .Subbarao and A. Nikzad, "Focusing techniques," Journal of Optical Engineering, vol. 32 (11), pp. 2824-2836, 1993.

[5] A.P. Pentland, "A new sense of depth of field," IEEE Transactions on Pattern Analysis and Machine Intelligence, vol. 9, no. 4, pp. 523-531, jul 1987.

[6] D.T. Pham and V. Aslants, "Depth from defocus using a neural network," Pattern Recognition, vol. 32, pp. 715-727, 1999

[7] M. Subbarao, "Parallel depth recovery by changing camera parameters," in Proc. Second International IEEE Conference on Computer Vision, Tampa, Florida, USA, 1988, pp. 149-155.

[8] M. Subbarao and G. Surya, "Depth from defocus : A spatial domain approach," International Journal of Computer Vision, vol. 13, no. 3, pp. 271-294, 1994.

[9] C.S. Williams and A.A. Becklund, Introduction to the OTF, Wiley Series in Pare and Applied optics, 1989.

[10] M. Subbarao, "Determining distance from defocused images of simple objects," Tech. Rep. 89-07-20, Computer visoin laboratory, Dpt of Electrical Engineering, State University of New-York, Stony Brook, NY 117942350, USA, 1989.

[11] G. Schneider, Numerical Analysis of Monocular Depth Sensing Principles and their Applications in Robotics, Ph.D. thesis, IAR-Université de Nancy 1, CRAN, Université de Nancy 1, July 1995.

[12] A.P. Pentland, "Depth of scene from depth of field," in Proc. Image Understanding Workshop, 1982, pp. 253259.

[13] T. Simon, Appréciation de la Profondeur Depuis des Images Nette et Floue en Vision 3D Passive, Automatique, Université de Nancy 1, Juillet 1991. 